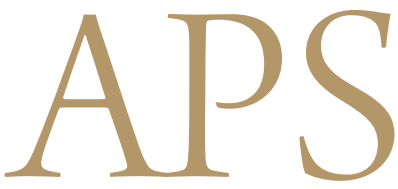

Archives of Plastic Surgery

\title{
Proximal Interphalangeal Joint Dislocations and Treatment: An Evolutionary Process
}

\author{
Kenneth Michael Joyce ${ }^{1}$, Cormac Weekes Joyce ${ }^{1}$, Frank Conroy ${ }^{2}$, Jeff Chan ${ }^{1}$, Emily Buckley ${ }^{2}$, \\ Sean Michael Carroll ${ }^{2}$ \\ ${ }^{1}$ Department of Plastic and Reconstructive Surgery, Galway University Hospital, Galway; ${ }^{2}$ Department of Plastic and Reconstructive Surgery, \\ St. Vincent's University Hospital, Dublin, Ireland
}

Background Proximal interphalangeal joint (PIPJ) dislocations represent a significant proportion of hand clinic visits and typically require frequent follow-ups for clinical assessment, orthotic adjustments, and physiotherapy. There are a large number of treatment options available for PIPJ dislocations, yet no prospective or controlled studies have been carried out, largely due to the diversity of the various types of injuries.

Methods We retrospectively reviewed all the PIPJ dislocations in our institution over a fiveyear period and directly compared the different splinting techniques that we have used over this time frame.

Results There were a total of 77 dislocations of the PIPJ (57 men and 20 women) that were included in our study. We found that our management has shifted gradually from complete immobilisation to controlled early mobilisation with figure-of-eight splints. Following treatment, the range of motion of the PIPJ in the figure-of-eight group was significantly greater than that in the other three methods $(P<0.05)$ used. There were significantly fewer hospital visits in the figure-of-eight splint group than in the other treatment groups.

Conclusions The treatment of PIPJ dislocations has undergone a significant evolution in our experience. Early controlled mobilisation has become increasingly important, and therefore, splints have had to be adapted to allow for this. The figure-of-eight splint has yielded excellent results in our experience. It should be considered for all PIPJ dislocations, but careful patient selection is required to achieve optimum results.

Keywords Finger joint / Dislocation / Splint

\author{
Correspondence: \\ Kenneth Michael Joyce \\ Department of Plastic and \\ Reconstructive Surgery, Galway \\ University Hospital, Galway, Ireland \\ Tel: +353879654586 \\ Fax: +35391542000 \\ E-mail: kennethjoyce1@gmail.com
}

Received: 31 Jan 2014 • Revised: 29 Mar 2014 • Accepted: 21 Apr 2014

pISSN: 2234-6163 • elSSN: 2234-6171 • http://dx.doi.org/10.5999/aps.2014.41.4.394 • Arch Plast Surg 2014;41:394-397

No potential conflict of interest relevant to this article was reported.

\section{INTRODUCTION}

Dislocations of the proximal interphalangeal joint (PIPJ) are the most common ligamentous injury of the hand [1-3] and can cause significant long-term pain, stiffness, and joint instability as well as post-traumatic arthrofibrosis [4]. These injuries can lead to absenteeism from work due to frequent hospital visits for medical therapy, occupational therapy, and/or physiotherapy. Management of these injuries requires a careful multidisciplinary approach that can be a heavy burden on increasingly precious hospital resources.

The complex biomechanics and anatomy of the PIPJ make it very vulnerable to post-traumatic pain and stiffness. A disruption of any of the important joint structures will impact the co- 
ordinated gliding motion of tendons and ligaments and impair the range of motion (ROM) of the joint [5].

Some authors advocate early, protected motion following simple PIPJ dislocations [6]. For this to occur, early presentation is ideal to reduce and prevent adhesion formation. There are a large number of treatment options available for PIPJ dislocations, yet no prospective or controlled studies have been carried out, largely due to the diversity of the various types of injuries [7]. Conservative measures include immobilisation, buddystrapping, dorsal-blocking splints, and figure-of-eight splints. The goal of PIPJ injury management is to obtain a strong, stable, and pain-free joint with an optimum ROM.

In our experience, simple PIPJ dislocations account for a significant portion of hand clinic visits and utilise a substantial amount of our resources. These injuries typically require one or more assessments by a hand surgeon as well as several reviews by the occupational and physical therapists over an extended period of time.

A significant reason behind poor outcome after PIPJ dislocations is due to adhesions, and the sites most vulnerable are the dorsal skin and the extensor apparatus. Frequently, a dislocation injury is often initially viewed as trivial, and this can often cause a delay in presentation. This may also lead to suboptimal management through prolonged immobilisation that contributes to long-term stiffness and poor function of the affected digit.

The aim of our study was to evaluate the outcome of PIPJ dislocations at St. Vincent's University Hospital, through a direct comparison of the different splinting techniques that have been used at our unit over several years. We hypothesised that figureof-eight splinting results in significantly fewer hospital visits than other conservative measures and provides better long-term results in terms of ROM when compared to other methods.

\section{METHODS}

All PIPJ dislocations treated at our institution from 2006 to 2012 were examined retrospectively. Any dislocation with an associated fracture was excluded from our study, as were interphalangeal dislocations of the thumb and PIPJ dislocations with central slip injuries. In total, 103 PIPJ dislocations without fractures were found in our database. Of these, complete clinical

\section{Table 1. Data representing the digits involved in the PIPJ} dislocations

\begin{tabular}{|lcccc|}
\hline Digit affected & Little & Ring & Middle & Index \\
\hline No. of patients (\%) & 26 & 22 & 15 & 14 \\
\hline
\end{tabular}

PIPJ, proximal interphalangeal joint. data were available on 77 patients $(61.2 \%)$. By reviewing medical records, details were recorded on each patient, including gender, age, hand dominance, hand affected, digit affected, mechanism of injury, time to presentation (time between initial injury and first medical visit to our institution), active PIPJ ROM, splinting method, and total number of hospital visits (medical, physiotherapy, and occupational therapy reviews combined). Various methods of splinting were compared in terms of PIPJ ROM at the end of treatment as well as the total number of hospital visits.

\section{Statistical analysis}

The Kolmogorov and Smirnov method was used to determine the normality of distribution of data. Bartlett's test was used to test the differences in standard deviations between groups. Log transformations of the total range of movement and the number of visits were carried out prior to one-way analysis of variance with Tukey-Kramer multiple comparisons. A P-value of $<0.05$ was considered statistically significant.

\section{RESUITS}

There were 77 dislocations of the PIPJ (57 men and 20 women) included in our study. The mean age of those who attended was 31.8 years. Sixty four patients were right-handed, and 13 were left-handed. The dominant hand was affected in 40 of these cases $(51.9 \%)$ and the non-dominant in 37 (48.1\%). The injury occurred in 55 patients $(71.4 \%)$ whilst playing sport. The digit most commonly affected was the little finger, which 26 patients injured (Table 1). The mean time from injury to presentation to our institution can be seen in Table 2. All patients were treated by one of the following four methods (Table 2): figure-of-eight splinting, dorsal blocking splinting, immobilisation, or buddystrapping (Fig. 1).

Following treatment, the ROM of the PIPJ (Table 3) in the figure-of-eight splint group was significantly greater than that in the other three groups $(\mathrm{P}<0.05)$. The total number of hospital visits was calculated by adding all the outpatient clinic visits to

Table 2. Data displaying the number of patients in each treatment group and the mean time to presentation in dayststandard deviation

\begin{tabular}{|lcc|}
\hline $\begin{array}{c}\text { Method of } \\
\text { treatment }\end{array}$ & $\begin{array}{c}\text { No. of } \\
\text { patients }\end{array}$ & $\begin{array}{c}\text { Time to } \\
\text { presentation (day) }\end{array}$ \\
\hline Figure-of-eight & 25 & $6.04 \pm 4.3$ \\
Dorsal blocking & 17 & $5.53 \pm 2.95$ \\
Buddy-strapping & 19 & $8 \pm 3.29$ \\
Immobilisation & 16 & $4.87 \pm 3.3$ \\
\hline
\end{tabular}




\section{Fig. 1. Photographs demonstrating the figure-of-eight splint}

(A) Dorsal view. (B) Lateral view. (C) Volar view.
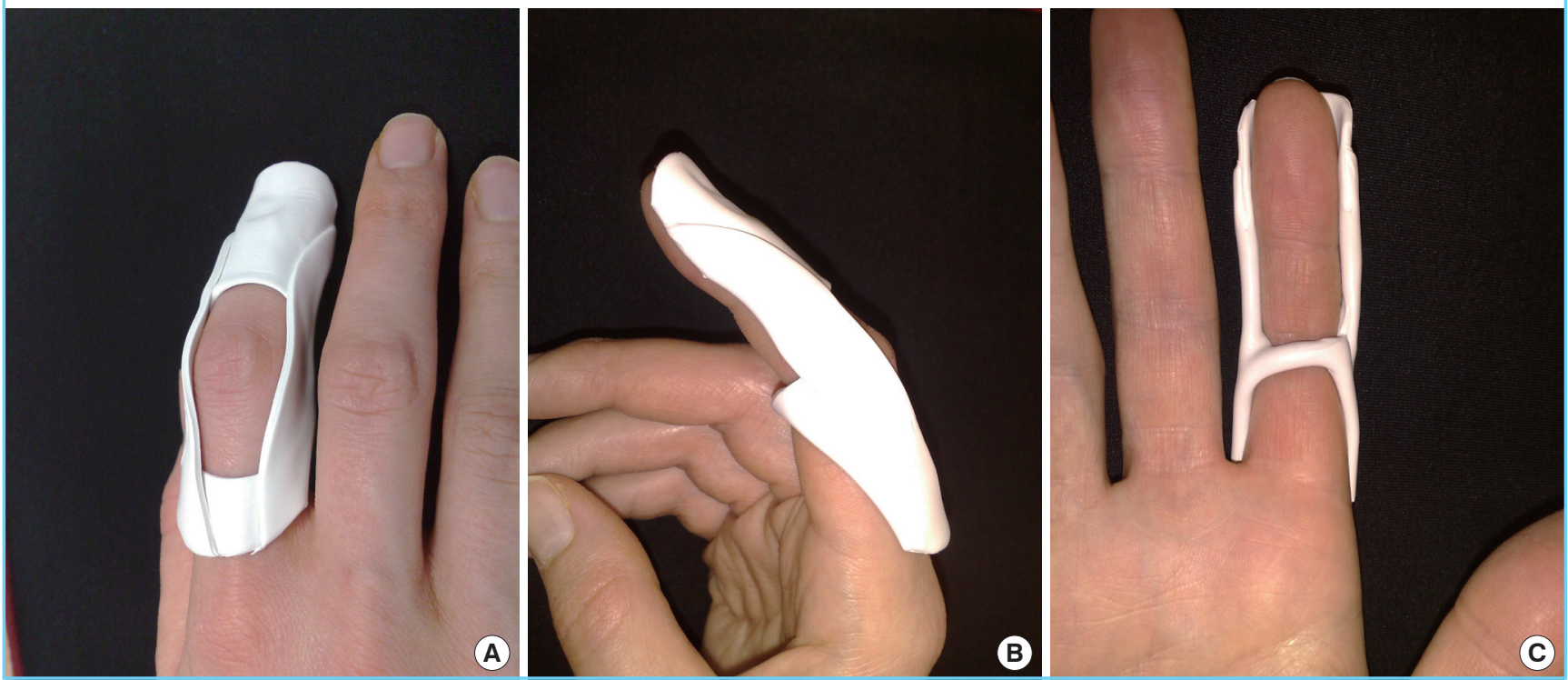

Table 3. Data showing ROM at the PIPJ post-treatment measured in degrees and standard deviation

\begin{tabular}{|lcc|}
\hline $\begin{array}{c}\text { Method of } \\
\text { treatment }\end{array}$ & $\begin{array}{c}\text { ROM } \\
\text { post-treatment }\left(^{\circ} \text { ) }\right.\end{array}$ & $\begin{array}{c}\text { P-value } \\
\text { (versus figure-of-eight) }\end{array}$ \\
\hline Figure-of-eight & $89.22 \pm 10.97$ & - \\
Dorsal blocking & $64.13 \pm 12.42$ & $<0.05$ \\
Buddy-strapping & $66.13 \pm 9.87$ & $<0.05$ \\
Immobilisation & $60.56 \pm 11.9$ & $<0.05$ \\
\hline
\end{tabular}

ROM, range of motion; PIPJ, proximal interphalangeal joint.

the number of physiotherapy and occupational therapy attendances. The average total number of hospital visits per patient was 5.6. The mean number of hospital visits for each treatment option is shown in Table 4. There were significantly fewer hospital visits in the figure-of-eight splint group than in the other treatment groups.

\section{DISCUSSION}

The cornerstone for optimum treatment of PIPJ dislocations is early detection and intensive rehabilitation [8]. Most PIPJ dislocations are amenable to non-operative treatment and recognised conservative measures include immobilisation, buddy strapping, dorsal blocking, and figure-of-eight orthoses. The trend in managing PIPJ dislocations has evolved over the years in response to our increased understanding of the physiological processes involved in healing as well as technological advances in splint materials.

The figure-of-eight splint is a custom-made thermoplastic
Table 4. Data showing the mean number of hospital visits for each treatment option and standard deviation

\begin{tabular}{|lc|}
\hline Method of treatment & Average no. of hospital visits \\
\hline Figure-of-eight & $3.2 \pm 2.19$ \\
Dorsal blocking & $6.9 \pm 3.94$ \\
Buddy-strapping & $6.8 \pm 3.85$ \\
Immobilisation & $8.1 \pm 2.8$ \\
\hline
\end{tabular}

splint that allows protected motion of the PIPJ. It is a light-weight material that provides good protection to the PIPJ during rehabilitation. It functions by providing support for the collateral ligaments and the volar plate [9] and eliminates further injury to the collaterals by preventing any ulnar or radial forces on the joint. The figure-of-eight splint blocks extension at $-30^{\circ}$ and prevents hyperextension at the PIPJ, thereby allowing the volar plate to heal if injured. However, it still allows for full active and passive flexion as well as limited active and passive extension. This prevents the joint from stiffening by allowing the central slip and flexor tendon to glide. Furthermore, patients report that this splint is comfortable to wear and that it protects the joint from further injury. They also find that the figure-of-eight splint is preferable to buddy-strapping as it provides more PIPJ support.

Early controlled mobilisation with the figure-of-eight splint has the potential to enhance the quality of PIPJ healing and a person's functional recovery. Early active or passive motion of one or both joints adjacent to a healing PIPJ dislocation in a figure-of-eight splint facilitates motion of the regional soft tissues and joints, thereby reducing the potential for developing joint stiffness, muscle weakness, and tendon glide tethering, which 
are all associated with prolonged immobilisation [10]. Historically, the mainstay in PIPJ dislocation treatment had been to completely immobilise the joint and sometimes the joints proximal and distal to it. Immobilization can contribute, along with the injury, to temporary and sometimes permanent limitations in a person's capacity to use the hand functionally. Healing connective tissues, including bone, have been shown to do better with functional or physiologic stress during the early stages of healing [11]. Early controlled mobilization of tendon, nerve, articular, and other hand injuries is considered part of good hand therapy practice [6].

A multi-disciplinary team approach to PIPJ dislocations is important as there is a large spectrum of injuries and many different approaches to their management. Close liaison between hand therapists and surgeons has certainly been a significant factor in the reduction of the number of hospital visits for patients with PIPJ dislocations in the recent years. The decisions of when and how to best control the motion require that the physician, therapist, and patient work together closely, and in harmony, with a complete understanding of the current strength or stability of the injured joint.

In our institution, we observed an obvious trend from 2008 onwards in the management of PIPJ dislocations. Prior to 2008, immobilisation was the preferred method of treatment. From 2008 to 2010, buddy strapping and dorsal blocking splints were the most frequently used options, and since 2010, the figure-ofeight splint has been the preferred method of splinting. We have demonstrated that the ROM at the PIPJ is significantly better in the figure-of-eight splint group than in the groups treated with traditional methods. This in turn has led to a substantial decrease in the number of hospital visits required for PIPJ dislocations.

As the spectrum of PIPJ injuries can range from mild ligamentous sprains to comminuted intra-articular fractures, no treatment method best suits all types. The figure-of-eight splint is most effective for volar plate and collateral ligament injuries of the PIPJ when there is no associated central slip injury. In our institution, it has become the preferred treatment option for simple PIPJ dislocations and previous methods of treatment, including immobilisation and dorsal blocking splints, have fallen out of favour. The figure-of-eight splint produces a significantly improved ROM post-treatment when compared to the alternative methods and leads to fewer hospital visits, which has a positive impact on the hospital budget.
The treatment of PIPJ dislocations has undergone a significant evolution in our experience. Early controlled mobilisation has become increasingly important, and therefore, the splints have had to adapt to allow for this. The figure-of-eight splint is one such tool in our armamentarium and has yielded excellent results in our experience. It should be considered for all PIPJ dislocations, but careful patient selection is required to achieve optimum results.

\section{REFERENCES}

1. Chinchalkar SJ, Gan BS. Management of proximal interphalangeal joint fractures and dislocations. J Hand Ther 2003; 16:117-28.

2. Deshmukh NV, Sonanis SV, Stothard J. Irreducible volar dislocations of the proximal interphalangeal joint. Emerg Med J 2005;22:221-3.

3. Kannan RY, Wilmshurst AD. Unstable proximal interphalangeal joint dislocations: another cause. Emerg Med J 2006;23:819.

4. Glickel SZ, Barron OA. Proximal interphalangeal joint fracture dislocations. Hand Clin 2000; 16:333-44.

5. Minamikawa Y, Horii E, Amadio PC, et al. Stability and constraint of the proximal interphalangeal joint. J Hand Surg Am 1993;18:198-204.

6. Feehan LM, Tang CS, Oxland TR. Early controlled passive motion improves early fracture alignment and structural properties in a closed extra-articular metacarpal fracture in a rabbit model.J Hand Surg Am 2007;32:200-8.

7. Freiberg A. Management of proximal interphalangeal joint injuries. Can J Plast Surg 2007;15:199-203.

8. Bailie DS, Benson LS, Marymont JV. Proximal interphalangeal joint injuries of the hand. Part I: anatomy and diagnosis. Am J Orthop (Belle Mead NJ) 1996;25:474-7.

9. Laporte JM, Berrettoni BA, Seitz WH Jr, et al. The figure-ofeight splint for proximal interphalangeal joint volar plate injuries. Orthop Rev 1992;21:457-62.

10. Latta LL, Sarmiento A, Tarr RR. The rationale of functional bracing of fractures. Clin Orthop Relat Res 1980;(146):2836.

11. Cyr LM, Ross RG. How controlled stress affects healing tissues. J Hand Ther 1998;11:125-30. 